\title{
Thalidomide in Axial Spondyloarthropathy: An Eastern Indian Experience
}

\author{
${ }^{1}$ Rudrajit Paul, ${ }^{2}$ Arijit Sarkar, ${ }^{3}$ Chandan K Das, ${ }^{4}$ Urmimala Bhattacharjee, ${ }^{5}$ Ritasman Baisya, ${ }^{6}$ Pallab Biswas, \\ ${ }^{7}$ Rathindranath Sarkar
}

\begin{abstract}
Background: Axial spondyloarthropathy $(\mathrm{SpA})$ is seronegative arthritis which mainly affects young adults and causes much morbidity. Tumor necrosis factor-alpha (TNF- $\alpha$ ) is one of the main mediators of inflammation in joints and enthuses in SpA. Nonsteroidal anti-inflammatory drugs (NSAIDs), diseasemodifying antirheumatic drugs (DMARDs), and biologics like TNF- $\alpha$ inhibitors are the mainstay of therapy in SpA. However, in India, the cost is one limiting factor in the use of biologics for the treatment of SpA in eligible patients. Thalidomide is an oral TNF- $\alpha$ inhibitor which has reported benefit in axial SpA, and it is a cheap option in the Indian setting. However, there is almost no Indian data on its efficacy.
\end{abstract}

Materials and methods: This is a retrospective analysis of patients receiving thalidomide for axial $\mathrm{SpA}$ in the rheumatology clinic of a tertiary care hospital of Kolkata. In this clinic, thalidomide was used at $100 \mathrm{mg} /$ day. Patients receiving thalidomide, who had completed at least one year follow up were included in the analysis. Assessment of SpondyloArthritis International Society (ASAS)-20, ASAS-40, and ankylosing spondylitis disease activity score with C-reactive protein (ASDAS-CRP) were measured three monthly during the follow-up. Side effect profile of thalidomide was also recorded.

Results: The completed data of 66 patients were analyzed. All patients were human leukocyte antigen (HLA) B27 positive. Ninety-five point percent fulfilled ASAS-20 criteria at 12 months and $71.2 \%$ fulfilled the ASAS- 40 criteria at 12 months. Three patients were nonresponders. The average ASDAS-CRP score also decreased from 2.9 to 1.5 over one year.

Conclusion: In our study, oral thalidomide resulted in significant improvements in axial SpA. Further randomized controlled

\footnotetext{
${ }^{1}$ Associate Professor, ${ }^{2}$ Demonstrator, ${ }^{3-6}$ Resident, ${ }^{7}$ Ex-Professor and $\mathrm{HOD}$

${ }^{1}$ Department of Medicine, Midnapore Medical College, Paschim Medinipur, West Bengal, India

${ }^{2}$ Department of Medical Microbiology, Institute of Post-Graduate Medical Education and Research and Seth Sukhlal Karnani Memorial Hospital, Kolkata, West Bengal, India

${ }^{3-6}$ Department of Medicine, Medical College Kolkata, West Bengal, India

${ }^{7}$ Department of Medicine, Medical College Kolkata, West Bengal, India

Corresponding Author: Rudrajit Paul, Associate Professor Department of Medicine, Midnapore Medical College, Paschim Medinipur, West Bengal, India, Phone: +919433824341, e-mail: docr89@gmail.com
}

trials are needed to find the relative efficacy of thalidomide with respect to other forms of treatment.

Keywords: Ankylosing, Spondylarthropathies, Spondylitis, Thalidomide, Tumor necrosis factor-alpha.

How to cite this article: Paul R, Sarkar A, Das CK, Bhattacharjee U, Baisya R, Biswas P, Sarkar R. Thalidomide in Axial Spondyloarthropathy: An Eastern Indian Experience. Bengal Physician Journal 2018;5(2):3-6.

\section{Source of support: Nil}

\section{Conflict of interest: None}

\section{INTRODUCTION}

The SpA is a heterogeneous group of disorders comprising of ankylosing spondylitis (AS) as the prototype with psoriatic arthritis, reactive arthritis and other lesser known entities in addition. ${ }^{1}$ According to the ASAS, SpA can be broadly divided into two categories: predominantly axial and predominantly peripheral. This is one of the causes of chronic low back pain in young adults and causes significant morbidity. Although early treatment often brings significant benefit, SpA is frequently diagnosed late, in part because of the lack of awareness among the physicians. The exact prevalence of SpA is not known, but according to a recent estimate, it affects 0.9 to $1.4 \%$ of the adult population in the USA. ${ }^{1}$ The economic costs of the treatment of SpA are huge and indirect costs due to functional disability are often higher than the actual treatment cost. ${ }^{2}$ Thus, early diagnosis and treatment of this entity are essential to reduce the societal burden and improve the quality of life of the individual patient.

The pathogenesis of SpA is complex. This is not explained by one single factor, but instead, there is a complex interplay of susceptibility genes, environmental triggers, and overactivation of the innate immune system. ${ }^{3}$ One of the most well-known genetic links to SpA is HLA-B27. ${ }^{4}$ The exact pathogenetic role of this major histocompatibility complex (MHC) is not known, but it is said to bind and present some arthritogenic peptides which can activate the CD8+ cells and lead to inflammation. ${ }^{4}$ There is another hypothesis that protein misfolding of some HLA-B27 subtypes may trigger a pathway known as unfolded protein response (UPR) which may then activate the proinflammatory NF-kB pathway. ${ }^{4}$ Some specific cytokines are important in SpA, and two of them are TNF- $\alpha$ and IL-17. 
Tumor necrosis factor- $\alpha$ (TNF- $\alpha$ ) is one of the main links in the pathogenesis of SpA. ${ }^{5} \mathrm{TNF}-\alpha$ is a pleiotropic cytokine. It induces inflammation itself and also activates other pathways of inflammation..$^{5}$ Levels of TNF- $\alpha$ are raised both in the serum and synovium in SpA patients. ${ }^{6}$ Thus, inhibition of TNF- $\alpha$ is one of the main therapeutic pathways in SpA. ${ }^{5}$

Current (2015) guidelines for the treatment of SpA advocates the use of NSAIDs, DMARDs (disease modifying anti-rheumatic drugs) and biologic agents. ${ }^{7}$ For the peripheral variety of $\mathrm{SpA}$, DMARDs like sulphasalazine, leflunomide and methotrexate are quite effective, but these are grossly ineffective for the axial variety. Thus, for axial SpA, biologics like anti-TNF or anti-IL-17 are the mainstay of therapy. Among the biologic agents, antiTNF agents are considered as the first line and strongly recommended for patients with inadequate response to or contraindication/intolerance to NSAIDs. ${ }^{7}$ A recent Cochrane review also concluded that anti-TNF agents significantly improve clinical symptoms in AS and the toxicity profile appears acceptable. ${ }^{8}$ However, in a country like India, where a large part of the health expenses is out-of-pocket, the cost is an important limiting factor in the guideline-based use of anti-TNF biologicals.

Thalidomide, a derivative of glutamic acid, is known to block TNF- $\alpha .{ }^{9}$ It has been tried in the treatment of axial SpA. ${ }^{9}$ Reports from other countries have demonstrated promising results, in terms of improvement of clinical symptoms, decrease in serum inflammatory markers and decrease in cytokine levels. ${ }^{9}$ Side effects are often a limiting factor in the use of thalidomide, but lower doses can be used to reduce the risk of such toxicity.

There are almost no major Indian studies concerning thalidomide in AS. However, studies from other countries on Asian patients have demonstrated significant efficacy. ${ }^{10}$ Since thalidomide is a low-cost option in the Indian setting, its use may be a potential way to lower the economic burden of biologic agent use.

The present study reports the retrospective data on thalidomide use in axial SpA in a rheumatology clinic from Eastern India.

\section{MATERIALS AND METHODS}

The present study is a retrospective data analysis of SpA patients attending the Rheumatology clinic of a tertiary care hospital in Kolkata. Data of SpA patients who were receiving thalidomide as per standard guidelines between June 2015 and 2016 were retrieved and analyzed. Since this was a record analysis, an expedited review was deemed sufficient by the institutional review board. Any patient who had received biologics or steroids was excluded from the analysis. For inclusion in the analysis, sequential data of the first year of treatment was used. Anyone with gaps in this 3-monthly follow-up was excluded. In this rheumatology clinic, patients with inflammatory low back pain were diagnosed with SpA as per recent standard criteria. ${ }^{11}$ HLA-B27 was checked in all patients. SpA patients were first started on NSAIDs and those who failed NSAID after 3 or more months were considered for DMARDs. Those patients who were started on thalidomide were first explained about the drug profile and probable adverse events as part of routine pre-treatment counseling. The written advice was provided for contraception, for both males and females.

As per the routine clinical protocol of this clinic, SpA patients on DMARD were followed up every three months (or earlier of needed). At each visit, clinical examination was done and ASDAS-CRPs Disease Activity Score), total spinal pain on a visual analog scale, duration of morning stiffness, patients' global assessment and bath ankylosing spondylitis functional index (BASFI) were documented. These were used to calculate the ASAS-20 and ASAS- 40 criteria. Blood tests for liver function test and hemogram were done at each visit. In this clinic, thalidomide was used as a standalone DMARD, and other drugs like sulfasalazine or methotrexate were not used with it. NSAIDs were continued as needed. None of the patients were on steroids.

Thalidomide dose was $50 \mathrm{mg}$ in the beginning (given at bedtime), subsequently increased to $100 \mathrm{mg}$ od after 2 to 4 weeks.

The data was first checked for normalcy. The degree of statistical significance between the values were analyzed by the paired ' $\mathrm{T}$ ' test. Correlation between parameters was assessed by calculating Spearman's rank correlation coefficient. Comparison of a number of individual reaching a certain degree of improvement was calculated by the chi-square test. A p-value $<0.05$ were considered significant.

\section{RESULTS}

In the aforementioned time period, complete data of 66 patients, who had received thalidomide for one year, could be retrieved. They had a mean age of $30.55 \pm 7.7$ years (range 20 to 50 years) and mean disease duration of $1.7 \pm 1.1$ years (range 6 month to 3 years) (Table 1 ). All the patients were HLA-B27 positive.

Table 1. Demographics of patients completing the study

\begin{tabular}{|c|c|c|}
\hline \multicolumn{2}{|c|}{ Age in years, mean (range) } & $30.55(20-50)$ \\
\hline \multicolumn{2}{|l|}{ Males (percentage) } & $42(63.6 \%)$ \\
\hline \multicolumn{2}{|l|}{ Females (percentage) } & $24(36.4 \%)$ \\
\hline \multicolumn{2}{|c|}{ Disease duration in years, mean (range) } & $1.7 \pm 1.1(0.5-3)$ \\
\hline \multirow{4}{*}{$\begin{array}{l}\text { Drug treatment: Past } \\
\text { drug therapy (before } \\
\text { thalidomide) }\end{array}$} & NSAIDs & 66 \\
\hline & Sulfasalazine & $28(42.4 \%)$ \\
\hline & Methotrexate & $14(21.2 \%)$ \\
\hline & Steroids & none \\
\hline
\end{tabular}


Table 2: Outcome measures in 66 patients included in the analysis ( $p$-value is shown for comparison with baseline)

\begin{tabular}{lllllll}
\hline Primary measures & & Baseline (0 months) & 3 months & 6 months & 9 months & 12 months \\
\hline ASDAS-CRP & Mean & 2.9 & 2.5 & 2.1 & 1.7 & 1.5 \\
& p-value & & $<0.001$ & $<0.001$ & $<0.001$ & $<0.001$ \\
BASFAI & Mean & 65.8 & 47.3 & 39.1 & 33.6 & 30.1 \\
& p-value & & $<0.001$ & $<0.001$ & $<0.001$ & $<0.001$ \\
Total spinal pain & Mean & 9 & 5.8 & 4.2 & 3.5 & 2.8 \\
& p-value & & $<0.001$ & $<0.001$ & $<0.001$ & $<0.001$ \\
Patient global assessment & Mean & 9 & 5.5 & 3.9 & 3.1 & 2.3 \\
& p-value & & $<0.001$ & $<0.001$ & $<0.001$ & $<0.001$ \\
Duration of morning stiffness \\
(minutes) & Mean & \multirow{2}{*}{80} & 52 & 31 & 27 & 22 \\
& p-value & & $<0.001$ & $<0.001$ & $<0.001$ & $<0.001$ \\
\hline
\end{tabular}

For this analysis, we had considered ASAS-20 and ASAS-40 improvement criteria (20\% and $40 \%$ improvement respectively in 3 out of 4 following domains, without worsening in the remaining domain, BASFI, morning stiffness, patient global assessment, pain) for determining positive treatment response. For the 66 patients who completed the 12-month course of thalidomide, $3(4.5 \%$, 95\% C.I.: 0.9 to $12.7 \%$ ) were nonresponders.

$36(54.5 \%)$ patients fulfilled ASAS-20 improvement criteria by 3 months. Fifty-one $(77.2 \%)$ patients achieved this goal by 6 months, and $58(87.8 \%)$ patients achieved response by 9 months. Sixty-three (95.5\%) patients fulfilled ASAS-20 by 12 months. ASAS 40 improvement criteria were fulfilled in $23(34.8 \%)$ patients in 3 months, in $35(53.1 \%)$ patients at 6 months, in $42(63.6 \%)$ patients at 9 months, and $47(71.2 \%)$ patients at 12 months (Table 2). Figure 1 schematically represents the trend of ASAS-20 and 40 over one year.

Gradually improving disease activity and amelioration of clinical symptoms was also demonstrated by a continuous decline in ASDAS-CRP score (Fig. 2).

\section{Adverse Effects}

Drop out because of adverse effects was recorded in 3 out of the 66 cases. These were because of peripheral

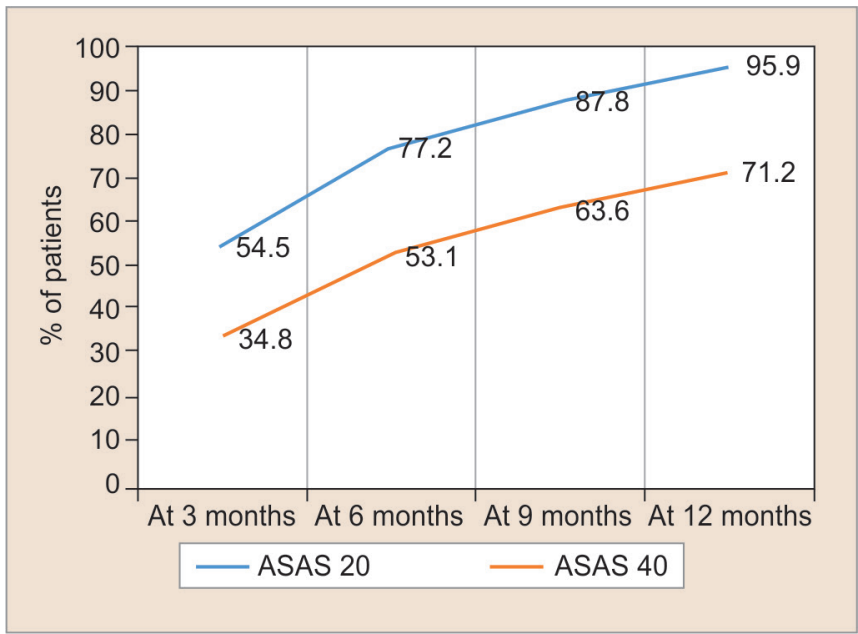

Fig. 1: Trend of the percentage of patients fulfilling ASAS-20 and ASAS-40 criteria neuropathy, constipation and gastric intolerance one each respectively. All the patients were evaluated with complete hemogram and liver function tests during each 3-monthly visit, and there was no significant change during the one year period. As peripheral neuropathy is a common side effect of thalidomide, all the patients were questioned regarding the symptoms of peripheral neuropathy like tingling and paresthesia of extremities however no positive response was recorded. Fatigue was a common symptom reported by $58(87.1 \%)$ of the subjects. But 38 (57.6\%) had fatigue before the commencement of thalidomide, probably as a result of secondary fibromyalgia in SpA.

\section{DISCUSSION}

In this retrospective data analysis from one rheumatology unit, it is seen that oral thalidomide use resulted in significant improvements in disease activity in SpA and this benefit was sustained over one year.

There are very few adequately powered studies assessing the efficacy of thalidomide in SpA. One prospective study was done in China by Huang et al. to assess the efficacy of thalidomide in SpA over one year ${ }^{10}$ In this, the target dose of thalidomide was $200 \mathrm{mg}$ while in our rheumatology clinic, the standard dose was $100 \mathrm{mg}$. similar to our study, Huang et al. also used thalidomide

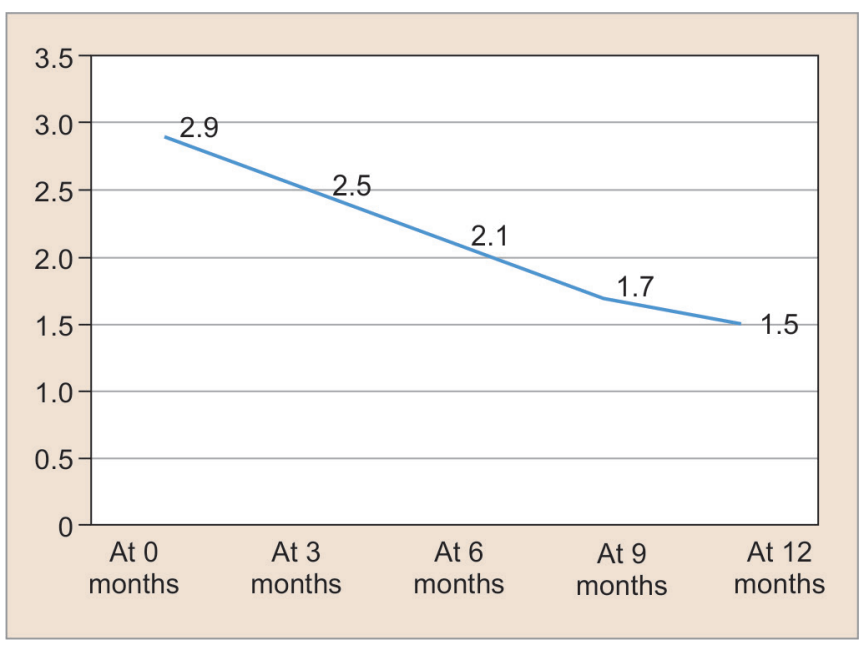

Fig 2: Schematic representation of gradual decline of ASDASCRP with thalidomide 
as the only DMARD, with or without NSAIDs. ${ }^{10}$ In this Chinese study, it was seen that thalidomide resulted in significant improvements in disease activity parameters at 3, 6 and 12 months. But when thalidomide was stopped at 12 months, the bath ankylosing spondylitis disease activity index (BASDAI) and BASFI scores deteriorated significantly after 3 months. However, the pain scores did not deteriorate. ${ }^{10}$ In our clinic, thalidomide was not stopped to assess the residual effect. Hence we cannot comment on this post-treatment effect. Similar to our data, the Chinese study also did not report any patient withdrawing due to adverse reactions of thalidomide, even at $200 \mathrm{mg} /$ day dose. Minor side effects like drowsiness resolved spontaneously.

Another open-label six-months' trial was done on AS patients in Taiwan. ${ }^{12}$ Here also, thalidomide was used at $200 \mathrm{mg} /$ day dose. The total response rate was $80 \%$ with significant improvements in BASDAI and BASFI at 6 months. ${ }^{12}$ There was also a significant decrease in the ESR. ${ }^{12}$ In our study also, as seen from Table 2, BASFI improved by more than $50 \%$ at 12 months. We did not check the BASDAI scores in our clinic.

In 2016, Islam et al. conducted a study on the treatment of juvenile idiopathic arthritis (JIA) with thalidomide. ${ }^{13}$ At 12 months, there was a 73\% improvement in active arthritis. ${ }^{13}$ Even there was a complete regression of hepatosplenomegaly and lymphadenopathy. This is similar to the Indian experience with JIA. ${ }^{14}$ Thus, thalidomide has shown efficacy in a related group of connective tissue disorders.

In 2013, Deng et al. showed that thalidomide reduced the risk of recurrence in AS. ${ }^{15}$ The recurrence rate was found to be low (compared to sulfasalazine and NSAIDs) in patients who received thalidomide following etanercept. Thus, the use of thalidomide may have a prognostic significance too in the management of AS.

Our study is limited by its retrospective design, a small number of patients, lack of measurement of serum cytokines levels like TNF- $\alpha$ and a short follow-up one year.

However, still, our study shows that similar to Chinese and Bangladeshi data, thalidomide was quite effective in Indian patients with negligible side effects. Thus, based on the available data, it can be said that thalidomide is effective in Asian patients. A further multi-center randomized controlled trial may be done to assess the efficacy of this treatment and generate a better quality of evidence.

\section{CONCLUSION}

Thalidomide is effective for axial SpA in Indian patients. The side effect profile is also acceptable. This can be a viable option for the Indian scenario when the cost of biologics is prohibitive.

\section{REFERENCES}

1. Taurog JD, Chhbra A, Colbert RA. Ankylosing Spondylitis and axial spondyloarthritis. N Engl J Med 2016;374:2563-2574.

2. Palla I, Trieste L, Tani C, Talarico R, Cortesi PA, Mosca M, et al. A systematic literature review of the economic impact of ankylosing spondylitis. Clin Exp Rheumatol. 2012;30:S136S141.

3. Ronneberger M, Schett G. Pathophysiology of spondyloarthritis. Curr Rheumatol Rep. 2011;13:416-420.

4. Shamji MF, Bafaquh M, Tsai E. The pathogenesis of ankylosing spondylitis.Neurosurg Focus. 2008;24:E3.

5. Davis JC Jr. Understanding the role of tumor necrosis factor inhibition in ankylosing spondylitis. Semin Arthritis Rheum. 2005;34:668-677.

6. Keller C, Webb A, Davis J. Cytokines in the seronegative spondyloarthropathies and their modification by TNF blockade: a brief report and literature review. Ann Rheum Dis 2003;62:1128-1132.

7. Ward MM, Deodhar A, Akl EA, Lui A, Ermann J, Gensler LS, et al. American College of Rheumatology/Spondylitis Association of America/Spondyloarthritis Research and Treatment Network 2015 Recommendations for the Treatment of Ankylosing Spondylitis and Nonradiographic Axial Spondyloarthritis. Arthritis Care Res (Hoboken). 2016;68: 151-166.

8. Maxwell LJ, Zochling J, Boonen A, Singh JA, Veras MMS, Tanjong Ghogomu E et al. TNF-alpha inhibitors for ankylosing spondylitis. Cochrane Database Syst Rev. 2015 Apr 18;(4):CD005468.

9. Braun J, Sieper J. Therapy of ankylosing spondylitis and other spondyloarthritides: established medical treatment, antiTNF- $\alpha$ therapy and other novel approaches. Arthritis Res. 2002;4:307-321

10. Huang F, Gu J, Hang J, Yu DTY. One year open label trial of thalidomide in ankylosing spondylitis. Arthritis Care and Research 2002;47:249-254.

11. Akgul O, Ozgocmen S. Classification criteria for spondyloarthropathies. World J Orthop. 2011;2:107-115.

12. Wei JC, Chan TW, Lin HS, Huang F, Chou CT. Thalidomide for severe refractory ankylosing spondylitis: A 6-month openlabel trial. J Rheumatol. 2003;30:2627-2631.

13. Islam MM, Islam MI, Talukdar MK, Haque M, Rahman SA. Efficacy and Safety of Thalidomide as Adjunct Therapy in Refractory Systemic Juvenile Idiopathic Arthritis Patients. Bangladesh Medical Research Council Bulletin 2016;42: 49-52.

14. Sathe K, Khubchandani RP. Thalidomide for systemic onset juvenile idiopathic arthritis. Indian Pediatrics. 2013 Feb 1;50(2):237-239.

15. Deng X, Zhang J, Zhang J, Huang F. Thalidomide reduces recurrence of ankylosing spondylitis in patients following discontinuation of etanercept. Rheumatol Int. 2013;33:14091413. 